Gynäkologische Endokrinologie 2009 · 7:80-86 DOI 10.1007/s10304-008-0299-9

Online publiziert: 5. April 2009

(c) Springer Medizin Verlag 2009

Redaktion

K. Diedrich, Lübeck

M.K. Bohlmann, Lübeck

\author{
M. Nitzschke ${ }^{1} \cdot$ T. Strowitzki ${ }^{2} \cdot$ M. von Wolff ${ }^{1}$ \\ ${ }^{1}$ Abteilung Gynäkologische Endokrinologie und Reproduktionsmedizin, \\ Universitätsklinik für Frauenheilkunde, Bern \\ ${ }^{2}$ Abteilung Gynäkologische Endokrinologie und \\ Fertilitätsstörungen, Universitäts-Frauenklinik, Heidelberg
}

\title{
Spermiogramm und Seminalplasma
}

\section{Der andrologische Faktor}

\begin{abstract}
Welche Information gibt uns das Spermiogramm über die "männliche“ Infertilität? Was bringt die neue IMSI-Technik? Wie können wir mit einfachen Maßnahmen das Befruchtungspotenzial unserer Patienten steigern, und wann empfehlen wir welche Behandlung? Welche Rolle spielt das Seminalplasma bei der Implantation? Sollte man Geschlechtsverkehr um die Zeit des Embryotransfers verbieten? Da diese Fragen dem Reproduktionsmediziner fast täglich in seiner Praxis begegnen, möchten wir im Folgenden die zugrunde liegende Evidenz darstellen und diskutieren.
\end{abstract}

\section{Spermiogramm - Grundlagen}

Die Anfertigung eines Spermiogramms gehört zu den wichtigsten Routineuntersuchungen im Rahmen der Abklärung von Kinderwunschpatienten. Die Aussagekraft von Spermiogrammen ist jedoch beschränkt, da es sich um eine stark untersucherabhängige und wenig standardisierte Methode handelt. Es ist daher von größter Wichtigkeit, die Samenanalysen möglichst in einem Labor mit viel Erfahrung auf diesem Gebiet durchführen zu lassen. Außerdem sollte sich das Labor möglichst regelmäßig internen und externen Qualitätskontrollen unterziehen. Da die Samenqualität bei einigen Männern stark schwanken kann, sollten immer 2 Spermiogramme in einem Abstand von
1-3 Wochen in dem gleichen Labor angefertigt werden. Bei der Auswertung sollten standardisierte Methoden zur Anwendung kommen, wobei in den meisten Fällen nach den von der WHO herausgegebenen Richtlinien untersucht wird [29].

Spermiogramm - was ist physiologisch, was pathologisch?

Für den Reproduktionsmediziner im klinischen Alltag ist die Beurteilung der Spermienkonzentration, der Spermienmotilität und der Spermienmorphologie zur groben Einschätzung der Spermienqualität eines Patienten besonders wichtig.

Die gängigen Normen der WHO erfüllen jedoch nur bedingt moderne klinische, technische und statistische Standards. Daraus folgte, dass in der neuesten Version des WHO-Manuals zur Samenanalyse [29] die Nomenklatur nicht mehr von Normalwerten spricht, sondern nur noch sog. Referenzwerte angibt.

Um herauszufinden, worin sich fertile von subfertilen Männern im Spermiogramm unterscheiden und wo die statistischen Grenzen gezogen werden können, untersuchten David et al. [6] im Rahmen einer groß angelegten Studie jeweils $2 \mathrm{Sa}$ menproben von 765 Männern aus ungewollt kinderlosen Partnerschaften sowie jeweils 2 weitere Proben von 696 Männern mit Kindern. Die subfertilen Männer wurden dabei aus 9 Kinderwunschzentren in den USA rekrutiert. Ihre Part- nerinnen waren in allen Abklärungen bezüglich Infertilität unauffällig, und im Durchschnitt bestand der unerfüllte Kinderwunsch bereits seit 43 Monaten. Die Kontrollgruppe der fertilen Männer wurde aus Pränatalkursen derselben Kliniken rekrutiert.

In ihrer Population hatten subfertile Männer eine Spermienkonzentration von weniger als 13,5 Mio./ml, weniger als $32 \%$ motile Spermien und weniger als $9 \%$ morphologisch normale Spermien (strikte Kriterien nach Dr. Thinus Kruger). Fertile Männer hatten eine Spermienkonzentration von mehr als 48,o Mio./ml, mehr als $63 \%$ motile Spermien und mehr als $12 \%$ morphologisch normale Spermien (nach Kruger). Die Werte zwischen den angegebenen Grenzen wurden als „unbestimmte Fertilität“ definiert. Das Risiko für Infertilität stieg mit abfallenden Werten für die einzelnen Samenparameter. Zum Beispiel war für den Wert „Spermienkonzentration“ die Odds Ratio für Infertilität, verglichen mit der fertilen Population, 1,5 in der unbestimmten Gruppe und 5,3 in der subfertilen Gruppe. Dabei kam es bei allen 3 gemessenen Parametern zu einer großen Überlappung der Werte zwischen den fertilen und den subfertilen Männern. Obwohl jeder der 3 Parameter dabei helfen konnte, zwischen fruchtbaren und unfruchtbaren Männern zu unterscheiden, war keiner von ihnen statistisch entscheidend. Die größte Aussagekraft hatte in diesem Fall noch der Prozentsatz an morphologisch normalen Spermien. 
Tab. 1 Vergleich der klinischen Para-

meter zwischen konventioneller ICSI

und einem direkt darauf folgenden

IMSI-Zyklus bei denselben Patienten.

(Mod. nach [12])

\begin{tabular}{|llll} 
& ICSI & IMSI & p-Wert \\
\hline Anzahl Transfer & 125 & 125 & - \\
\hline Transferierte Embryos & 265 & 261 & $>0,05$ \\
$\begin{array}{l}\text { Klinische Schwanger- } \\
\text { schaftsrate (\%) }\end{array}$ & 2,4 & 37,6 & $<0,001$ \\
\hline Implantationsrate (\%) & 0,8 & 20,3 & $<0,001$ \\
Geburtenrate (\%) & 0 & 17,6 & $<0,001$ \\
\hline
\end{tabular}

In Anbetracht dieser Ergebnisse wird von den Autoren vorgeschlagen, nicht mehr wie bisher nur einen einzigen Wert für jeden gemessenen Parameter festzulegen, um zwischen normalem und pathologischem Spermiogramm zu unterscheiden. Stattdessen sollte man 2 Grenzwerte angeben und in die Gruppen „fertill“, „unbestimmt" und „subfertil“" unterteilen.

\section{Spermiogramm als Entscheidungs- grundlage für Therapieoptionen}

Bei einem andrologisch abgeklärten Patienten mit Kinderwunsch, der ein pathologisches Spermiogramm aufweist, stellt sich die Frage nach der geeigneten Behandlungsmethode. Grundsätzlich sind zur Behandlung der männlichen Infertilität die intrauterine Insemination, die IVFoder die ICSI-Behandlung möglich. Um zu entscheiden, welches Paar von welcher Behandlungsmethode am meisten profitieren kann, ist es wichtig, den Einfluss der einzelnen Parameter eines Spermiogramms auf die Erfolgsrate der jeweiligen Methode zu kennen.

Ombelet et al. [17] untersuchten im Rahmen einer retrospektiven Studie den Einfluss der Parameter „Gesamtzahl motiler Spermien“ und „Spermienmorphologie (strikte Kriterien nach Kruger)" auf den Ausgang von 792 mit Clomiphen stimulierten, intrauterinen Inseminationen. Dabei fanden sie heraus, dass keiner der beiden Parameter eine besondere Rolle spielte, solange mehr als 1 Mio. bewegliche Spermien bei ausreichender Morphologie inseminiert wurden. Bei einer Gesamtzahl von weniger als 1 Mio. beweglichen Spermien im Inseminat, sahen sie jedoch keine einzige Schwangerschaft, wenn weniger als $4 \%$ morphologisch normale Sper-

Gynäkologische Endokrinologie 2009· 7:80-86 DOI 10.1007/s10304-008-0299-9

C) Springer Medizin Verlag 2009

\section{Nitzschke $\cdot$ T. Strowitzki $\cdot$ M. von Wolff Spermiogramm und Seminalplasma. Der andrologische Faktor}

\section{Zusammenfassung}

Das Spermiogramm liefert als wenig standardisierte Methode nur grobe Informationen zur Diagnostik männlicher Subfertilität. Daher sollte außer bei hoch pathologischen Werten das Spermiogramm nicht als alleinige Entscheidungsgrundlage für die Therapie dienen. Therapeutisch können neben der IC$\mathrm{SI}$ (intrazytoplasmatische Spermieninjektion) bei einer männlichen Infertilität auch mit intrauterinen Inseminationen gute Ergebnisse erzielt werden. In Fällen, bei denen selbst die ICSI-Methode versagt, scheint die IMSI-Technik (intrazytoplasmatische, morphologische selektierte Spermieninjektion) vielversprechend zu sein. Unabhängig von der Behandlungsmethode ist es von entscheidender Bedeutung, eine möglichst gute Samenprobe zu gewinnen. Dabei ist es möglich, mit einfachen Methoden wie einer Verkürzung der Abstinenzzeit oder einer Probengewinnung zu Hause die Samenqualität entscheidend zu verbessern. Neueste Erkenntnisse zeigen, dass die Anwendung von Seminalplasma in der Reproduktionsmedizin das Endometrium rezeptiver macht und somit die Implantation des Embryos unterstützen kann. Auch Geschlechtsverkehr um den Zeitpunkt des Embryotransfers scheint die Behandlung positiv zu beeinflussen.

Schlüsselwörter

Spermiogramm · IMSI · Samenqualität · Seminalplasma $\cdot$ Endometrium

\section{Semen analysis and seminal plasma. The male factor}

\section{Abstract}

Semen analysis is routinely used to evaluate the male partner in infertile couples. Unfortunately, sperm measurements that discriminate between fertile and infertile men are not well defined. Treatment decisions should therefore not be based exclusively on semen analysis, except for those cases with very poor sperm parameters. Treatments such as intra-uterine insemination (IUI) or intracytoplasmic sperm injection (ICSI) can usually be performed with sufficient pregnancy rates. In couples in which even ICSI fails, intracytoplasmatic, morphogically selected sperm injection (IMSI) seems to be promising. Collecting a semen sample with high quality is always important for male patients in assisted reproduction technology (ART) programs. Sperm quality can be improved using simple methods, such as modifying sexual abstinence or collecting semen samples at home. There is new evidence that the use of seminal plasma in ART treatment may improve endometrial receptivity and consequently implantation. Sexual intercourse around the time of embryo transfer also seems to improve the clinical outcome of ART.

Keywords Semen analysis $\cdot$ IMSI $\cdot$ Sperm quality $\cdot$ Seminal plasma $\cdot$ Endometrium 
mien vorhanden waren. Bei $4 \%$ oder mehr morphologisch normalen Spermien hatten sie überraschend gute Schwangerschaftsraten von durchschnittlich 13,6\% pro Zyklus, wenn weniger als 1 Mio. bewegliche Spermien inseminiert wurden.

Auch Wainer et al. [28] aus Frankreich interessierten sich für die Frage, welchen Einfluss die Gesamtzahl beweglicher Spermien und der Prozentsatz an morphologisch normalen Spermien im Inseminat auf den Ausgang von intrauterinen Inseminationen haben. Dazu untersuchten sie im Rahmen einer retrospektiven Studie 2564 IUI-Zyklen. Sie fanden dabei eine signifikant geringere Schwangerschaftsrate, wenn weniger als 1 Mio. bewegliche Spermien inseminiert wurde. Die Spermienmorphologie für sich alleine genommen war kein Faktor, der in signifikanter Weise den Ausgang einer intrauterinen Insemination beeinflussen konnte. Wenn jedoch im Inseminat weniger als 30\% morphologisch normale Spermien (nach David et al. 1975, modifiziert nach Jouannet et al. 1988) vorhanden waren, wurden die Schwangerschaftsraten schon bei einer Gesamtzahl von weniger als 5 Mio. beweglichen Spermien im Inseminat deutlich geringer.

Die Autoren schließen daraus, dass bei einem Prozentsatz von weniger als 30\% morphologisch normalen Spermien im Inseminat (nach David) mindestens 5 Mio. motile Spermien vorhanden sein sollten, um die beeinträchtigte Spermienqualität zu kompensieren. Falls dieser Wert nicht erreicht werden kann, sollte man eine IVF-Behandlung empfehlen.

Iberico et al. aus Alicante [14] untersuchten den Ausgang von 1010 intrauterinen Inseminationen im Verhältnis zur Gesamtzahl motiler Spermien im Inseminat. Dabei bildeten sie die Gruppen >30 Mio., 20-30 Mio., 10-20 Mio., 510 Mio. und <5 Mio. und konnten eine kontinuierliche Abnahme der Schwangerschaftsraten von 15,3 , über $8,3,8,2,5,2$ auf $3,6 \%$ in den jeweiligen Gruppen beobachten.

Van Voorhis et al. [25] von der University of Iowa interessierten sich für die Frage, ab welchem Wert für die Gesamtzahl an motilen Spermien im Nativspermiogramm der Kosten-Nutzen-Faktor für IVF/ICSI den einer intrauterinen In- semination übersteigt. Dafür werteten sie 3479 Inseminationszyklen und 551 IVF/ ICSI-Zyklen im Verhältnis zur Gesamtzahl motiler Spermien aus und kamen zum Schluss, dass bei weniger als 10 Mio. motiler Spermien im Nativspermiogramm IVF/ICSI im Verhältnis zur erzielten Schwangerschaftsrate für die Patienten unter Berücksichtigung aller Kosten in ihrem Zentrum kostengünstiger wurde als die intrauterine Insemination.

\section{(7) Es ist schwierig, allein auf der Basis des Spermiogramms eine Therapieentscheidung zu fällen}

Zusammenfassend kann gesagt werden, dass es in der Praxis sehr schwierig ist, allein auf der Basis des Spermiogramms eine Therapieentscheidung zu fällen. Außer bei hochpathologischen Spermiogrammen mit weniger als 1 Mio. mobilen Spermien kann es durchaus gerechtfertigt sein, dem Kinderwunschpaar bei alleiniger männlicher Indikation eine Insemination als erste Therapieoption anzubieten.

\section{Intrazytoplasmatische, morphologische selektierte Spermieninjektion}

Mit der Einführung von intrazytoplasmatischer Spermieninjektion (ICSI) glaubte man, das Problem männlicher Unfruchtbarkeit bis auf wenige Ausnahmen endgültig gelöst zu haben. Bis vor kurzer Zeit war man auch der Meinung, dass die Spermienmorphologie dabei nur wenig Einfluss auf die Resultate hatte. Erst eine Publikation von De Vos et al. aus dem Jahre 2003 [7] stellte einen Zusammenhang zwischen erfolgreicher ICSI-Behandlungen und der Spermienmorphologie her. Sie konnten zeigen, dass die Verwendung von morphologisch pathologischen, verglichen mit normalen Spermien eine geringere Befruchtungs- und Schwangerschaftsrate ergab. Andererseits ließ sich die Abortrate durch Behandlung mit morphologisch normalen Spermien signifikant verringern.

Inzwischen ist man der Auffassung, dass bei der Spermienmorphologie sog. Kernvakuolen eine wichtige Rolle spielen könnten. Ihr Vorhandensein scheint auf vermehrte Chromosomenschäden in den Spermien hinzudeuten. Franco et al. konnten in ihrer Arbeit aus dem Jahre 2007 [10] einen direkten Zusammenhang zwischen Kernvakuolen und DNASchäden aufzeigen. Dabei wurde ein TUNEL-Assey dazu verwendet, um vakuolisierte mit normalen Spermien zu vergleichen. Hierbei zeigte sich, dass in den vakuolisierten Spermien im Gegensatz zu unauffälligen Spermien sowohl der Prozentsatz an DNA-Fragmentationen (29 vs. $15,8 \%$ ) als auch der Prozentsatz an einzelsträngiger DNA (67,9 vs. $33 \%)$ signifikant erhöht waren.

Bei der ICSI-Technik werden die Spermien bei einer 200- bis 40o-fachen Vergrößerung unter dem Mikroskop ausgewählt. Dabei besteht die Möglichkeit, Anomalien des Zellkerns und insbesondere Vakuolen zu übersehen.

Mit der von Prof. Bartoov [3] entwickelten IMSI-Technik ist es nun möglich, mittels einer 600o-fachen Vergrößerung der Spermien in Echtzeit und ohne Färbung kleinste Vakuolen zu erkennen und somit untaugliche Spermien zu verwerfen. Damit soll die Wahrscheinlichkeit erhöht werden, ein genetisch intaktes Spermium ohne DNA-Schäden in die Eizellen zu injizieren. Er konnte mit Verwendung dieser Technik in einer FallKontroll-Studie mit jeweils 80 Paaren pro Gruppe, verglichen mit konventioneller ICSI, die Schwangerschaftsrate von 25 auf $60 \%$ steigern und gleichzeitig die Abortrate von 40 auf $14 \%$ reduzieren. Auch Hazout et al. [12] erzielten ähnliche Resultate mit der IMSI-Technik. Sie verglichen bei Patienten, die bereits 2 erfolglose Versuche mit der ICSI-Technik hinter sich hatten, die Ergebnisse eines neuen IMSI-Zyklus mit dem direkt vorangegangenen ICSI-Zyklus. Obwohl sie keine Unterschiede in den biologischen Parametern wie der Befruchtungsrate und der Embryomorphologie sahen, konnten die klinischen Parameter wie die Schwangerschaftsrate, die Implantationsrate und die Geburtenrate in ihrer Studie deutlich verbessert werden ( $\bullet$ Tab. 1).

Somit könnten ggf. Patienten mit einer schweren Teratozoospermie oder solche mit mehreren erfolglosen ICSI-Versuchen in der Vorgeschichte von der IMSITechnik profitieren. 


\section{Einfache Möglichkeiten zur Verbesserung der Spermienqualität}

Die Samenqualität kann als entscheidender Faktor den Ausgang von reproduktionsmedizinischen Behandlungen wie künstliche Inseminationen oder IVF beeinflussen. Daher ist es von entscheidender Bedeutung für jeden männlichen Patienten, eine möglichst gute Samenprobe abzugeben.

Es ist weltweit anerkannte Routine, die Samenproben für assistierte Reproduktion durch Masturbation zu gewinnen. Der Evolutionsbiologe Robin Baker [1] vertritt jedoch die These, dass die männliche Masturbation als ein Regulationsmechanismus dient, um die Samenqualität für den nächsten Geschlechtsverkehr zu optimieren. Daraus würde folgen, dass die Spermienqualität in durch Masturbation gewonnenen Samenproben nicht optimal wäre. Zavos et al. [30] konnten in der Tat eine deutliche Verbesserung der Samenqualität nachweisen, wenn das Sperma statt durch Masturbation mithilfe von Geschlechtsverkehr gewonnen wurde.

Sperma besteht aus zellulären Bestandteilen, den Samenzellen oder Spermien, und dem Sekret der akzessorischen Geschlechtsdrüsen, dem Seminalplasma. Der $\mathrm{pH}$-Wert von gesundem Sperma liegt im leicht sauren Bereich.

Sofikitis und Miyagawa [22] haben im Rahmen einer Studie Samenproben von 38 infertilen Männern untersucht, die jeweils 6 aufeinanderfolgende Proben durch Masturbation und dann 6 weitere durch Geschlechtsverkehr gewannen. Das Sperma wurde in einem nächsten Schritt auf die üblichen Samenparameter und auf die biochemischen Marker der Glandula vesicularis (Samenblasendrüse), Prostata und Ampulla ductus deferentis (Samenleiterampulle) hin untersucht. Neben einem deutlich erhöhten Seminalplasmavolumen fand man in den Proben, die durch Geschlechtsverkehr gewonnen wurden, auch die Spermienanzahl, ihre Motilität und den Prozentsatz an morphologisch normalen Spermien signifikant erhöht (- Tab. 2). Auch die Marker für die Prostatafunktion waren deutlich erhöht, wohingegen die Marker für die Sekretionsaktivität der Samenblasendrüse und der

Tab. 2 Vergleich der Samenparameter derselben Patienten je nach Methode der Probengewinnung. (Mod. nach [22])

\begin{tabular}{llll} 
& Masturbation & Geschlechtsverkehr & p-Wert \\
Anzahl Patienten & 38 & 38 & - \\
\hline Gesamtzahl Spermien (Mio.) & 44,3 & 99,4 & $<0,001$ \\
\hline Volumen (ml) & 2,3 & 3,3 & $<0,001$ \\
\hline Motile Spermien (\%) & 11,3 & 32,1 & $<0,001$ \\
Morphologisch normale Spermien (\%) & 32,3 & 41,3 & $<0,001$ \\
pH & 7,7 & 7,1 & $<0,001$ \\
\hline
\end{tabular}

Samenleiterampulle gleich blieben. Der signifikant verringerte $\mathrm{pH}$-Wert in den Samenproben nach Geschlechtsverkehr deutete ebenfalls auf eine erhöhte Prostatasekretion hin.

Die Autoren führten die bessere Spermienqualität nach Geschlechtsverkehr auf die erhöhte Prostataaktivität zurück, konnten jedoch nicht erklären, warum diese Drüse hierbei im Vergleich zu den anderen Anhangsdrüsen stärker aktiviert wird. In Anbetracht dieser Ergebnisse kann man davon ausgehen, dass eine durch Masturbation gewonnene Samenprobe für den jeweiligen Patienten nicht repräsentativ zu sein scheint. Ob diese Erkenntnis Einfluss auf die Samengewinnung in der klinischen Routine haben wird, bleibt offen.

\section{Einfluss der sexuellen Abstinenz auf die Samenqualität}

Die Dauer der sexuellen Abstinenz vor Kinderwunschbehandlungen, um die bestmögliche Samenqualität zu erhalten, ist ein viel diskutiertes Thema zwischen Kinderwunschärzten und Patienten. Unabhängig von der Samenqualität im Basisspermiogramm werden wohl die meisten Kinderwunschkliniken ihre männlichen Patienten dazu anhalten, die von der WHO empfohlene 2-7 Tage Abstinenz vor der Samenabgabe einzuhalten.

Levitas et al. [16] aus Israel hatten sich für die Frage interessiert, welchen Einfluss die sexuelle Abstinenz auf die einzelnen Parameter eines Spermiogramms hat. Sie untersuchten dazu in einer retrospektiven Studie 9689 Samenproben von 6008 Männern, die in ihrem Zentrum zwischen 1995 und 2003 zum Zwecke von Routineuntersuchungen oder für eine IUI-Behandlung gewonnen wurden. Gemäß den WHO-Richtlinien wurden 3506 Spermi- ogramme, die eine Spermienkonzentration von unter $20 \mathrm{Mio} . / \mathrm{ml}$ aufwiesen, als Oligozoospermie klassifiziert. Die restlichen 5983 Samenproben mit größer oder gleich $20 \mathrm{Mio} / \mathrm{ml}$ wurden als normozoosperm definiert. Alle Samenproben aus jeder Gruppe wurden dann nochmals in Bezug auf die jeweilige Abstinenzzeit aufgeteilt und statistisch untersucht.

Interessanterweise fanden sie in der oligozoospermen Gruppe mit zunehmender Abstinenzzeit eine stetige Abnahme morphologisch normaler Spermien sowie ihrer durchschnittlichen Motilität. Die beste Spermienqualität fanden sie nach einer Abstinenzzeit von nur wenigen Stunden bis zu 1 Tag. In der normozoospermen Gruppe hingegen stieg der Prozentsatz an motilen Spermien bis zum 7. Abstinenztag stetig an, und der Prozentsatz an morphologisch normalen Spermien blieb in den normozoospermen Proben bis zum 10. Abstinenztag stabil. Die gesamte Spermienanzahl und die Menge an beweglichen Spermien stiegen in der oligozoospermen Gruppe bis zum 4. Abstinenztag an und nahmen bei mehr Abstinenztagen wieder ab. Dieser Anstieg war in der normozoospermen Gruppe bis zum 7. Abstinenztag zu beobachten. Nach 11-14 Tagen fiel der Wert für die Anzahl an beweglichen Spermien hier isoliert wieder ab.

In Anbetracht ihrer Daten empfehlen die Autoren daher, bezüglich der Abstinenzzeit vor reproduktionsmedizinischen Behandlungen zwischen normozoospermen und oligozoospermen Männern zu unterscheiden. Patienten mit einer Oligozoospermie sollten vor Abgabe der Samenprobe höchstens $24 \mathrm{~h}$ abstinent bleiben, um einen möglichst hohen Prozentsatz an motilen und morphologisch normalen Spermien zu haben. Die Spermiengesamtzahl und die Anzahl an motilen Spermien können ggf. bis zum 4. Absti- 
nenztag gesteigert werden. Eine längere Abstinenz wäre in dieser Patientengruppe jedoch nicht zu empfehlen. Männer mit normalem Eingangsspermiogramm können ihre Spermienqualität mit einer Abstinenzzeit von bis zu 7 Tagen steigern. Mehr als 10 Tage Abstinenz sollten jedoch auch sie nicht überschreiten.

Eine andere israelische Gruppe um TurKaspa [24] untersuchte, wie sich die Samenparameter ändern, wenn man 2 kurz aufeinanderfolgende Ejakulate von demselben Mann miteinander vergleicht. Im Rahmen einer Fall-Kontroll-Studie wurden entsprechend jeweils 2 Samenproben von 576 Männern gewonnen und je nach Qualität des 1. Spermiogramms in 4 Gruppen eingeteilt. Die 2. Samenprobe wurde in der normozoospermen Gruppe, der asthenozoospermen Gruppe und einem Teil der oligozoospermen Gruppe (Gruppen 13) nach jeweils $24 \mathrm{~h} \mathrm{Abstinenz} \mathrm{gewonnen.}$ Ein anderer Teil der oligozoospermen Männer (Gruppe 4) gab die 2. Samenprobe bereits 1-4 h nach der 1 . Probe ab. In der Gruppe der 359 normozoospermen Männer sank die Gesamtzahl motiler Spermien erwartungsgemäß signifikant von durchschnittlich 93 Mio. auf 42 Mio. ab. Interessanterweise blieb jedoch in den anderen Gruppen die Gesamtzahl an motilen Spermien in der 2. Probe konstant oder stieg sogar leicht an. Alle Gruppen profitierten davon, 2 kurz aufeinanderfolgende Ejakulate zu poolen.

\section{Unterschiedliche Qualität des Samens nach Gewinnung durch Masturbation in der Klinik oder zu Hause}

Elzanaty und Malm [9] aus Schweden hatten sich für die Frage interessiert, ob es einen Unterschied macht, wo eine Samenprobe gewonnen wird. Sie untersuchten die Samenparameter von 379 Proben, die entweder in ihrem Zentrum oder zu Hause durch Masturbation gewonnen wurden. Alle Proben wurden innerhalb von 60 min nach der Abgabe untersucht, wobei die im Zentrum abgegebenen Proben im Durchschnitt 10 min früher analysiert wurden. Hierbei wurden keine Unterschiede im Volumen der Ejakulate oder der Spermienmorphologie gefunden. Auch die Zusammensetzung des Se- minalplasmas blieb in beiden Gruppen gleich. Die Autoren fanden jedoch signifikant bessere Werte für Spermienkonzentration (64 $\mathrm{zu} 41 \mathrm{Mio}$.) und schnell progressive Motilität (WHO A: $18 \mathrm{zu}$ 12\%) in den Proben, die zu Hause gewonnen wurden. Sie führten dieses Ergebnis v. a. auf den Faktor „Stress“ zurück. Auch Clarke et al. [4] hatten beobachtet, dass die Qualität einer Samenprobe von der Umgebung abhängig ist. Faktoren wie die Präsenz anderer Personen, Lage des Probengewinnungsraumes, hoher Geräuschpegel, Krankenhausatmosphäre, Platzmangel und das Fehlen der Partnerin während der Gewinnung einer Samenprobe, können zu Angst- oder Stressreaktionen beim Patienten führen und somit die Qualität der Probe negativ beeinflussen. Man kann davon ausgehen, dass in der schon zuvor erwähnten Studie zur Methode der Probengewinnung (Masturbation vs. Geschlechtsverkehr; [22]) die Proben in der Geschlechtsverkehrgruppe ebenfalls zu Hause gewonnen wurden. Auch hier waren die Samenparameter in der entsprechenden Gruppe deutlich verbessert. Daher ist zu überlegen, ob man nicht generell empfehlen sollte, eine Samenprobe, wenn möglich, zu Hause gewinnen zu lassen.

\section{Einfluss der Zeit zwischen Ejakulation und Untersuchung der Probe auf die Spermaqualität}

Elzanaty und Malm [8] untersuchten in einer anderen Studie den Einfluss des Zeitintervalls zwischen Gewinnung und Untersuchung einer Samenprobe auf seine Qualität. Die Ejakulate von 1079 Männern wurden nach einer durchschnittlichen Abstinenzzeit von 4 Tagen gesammelt, bei $37^{\circ} \mathrm{C}$ wurde die Verflüssigung abgewartet, um dann innerhalb von 24-180 min gemäß den WHO-Kriterien ausgewertet $\mathrm{zu}$ werden. Sie unterteilten 3 Gruppen von Zeitintervallen (24-30 min, 3160 min, 63-180 min). Die Spermienmorphologie und der Prozentsatz an lebenden Spermien unterschieden sich nicht signifikant zwischen den einzelnen Gruppen. Der Anteil an schnell progressiven Spermien (WHO-Motilität A) war jedoch in den Gruppen 31-6o min (14\%) und 63180 min (10\%) schon signifikant geringer als in der Gruppe $24-30$ min (17\%). Die gesamte Fraktion progressiver Spermien (WHO-Motilität A+B) war in der Gruppe $63-180$ min (46\%) im Vergleich zu den Gruppen 24-30 min (51\%) und 31-60 min (50\%), signifikant vermindert. Die Autoren schließen aus diesen Resultaten, dass Samenproben innerhalb von $60 \mathrm{~min}$ nach der Verflüssigung ausgewertet und verwendet werden sollten.

\section{Seminalplasma - ein Therapeutikum zur Verbesserung der Implantationsrate?}

Bei der IVF und ICSI beträgt die Implantationsrate selbst unter optimalen Voraussetzungen und unter Selektion der qualitativ besten Blastozysten nur ca. 50\%.

\section{- Es ist davon auszugehen, dass ein Teil der Implantationsversager auf einer endometrialen Dysregulation beruht.}

Diese Annahme wird gestützt durch die Arbeiten von Horcajades et al. [13], die bei einer hoch dosierten Gonadotropinstimulation eine Dysregulation zahlreicher endometrialer Faktoren nachgewiesen haben. Aufgrund dessen stellt sich die Frage, ob die Dysregulation des Endometriums behandelt werden kann.

Interessanterweise lassen Erkenntnisse aus Tierversuchen vermuten, dass die endometriale Rezeptivität durch die direkte Interaktion von Seminalplasma und Spermatozoen mit dem Endometrium beeinflusst wird. In-vivo-Versuche an Mäusen belegen eine Induktion morphologischimmunologischer Veränderungen des Endometriums vor der Implantation durch einzelne seminalplasmatische Zytokine, wie z. B. TGF- $\beta$ [20, 21]. So stimuliert TGF- $\beta$, das in hohen Konzentrationen im Seminalplasma der Maus und des Mannes $\mathrm{zu}$ finden ist, sowohl in vitro als auch in vivo die epitheliale Expression von GMCSF, das wiederum eine wesentliche Rolle bei der Entwicklung des Mäuseembryos zu spielen scheint $[19,20]$.

Auch im menschlichen Seminalplasma finden sich zahlreiche Zytokine wie IL-1 $\beta$, IL-2, IL- 6 und TGF- $\beta$, die aufgrund der uterinen Kontraktilität postkoital unter physiologischen Bedingungen in Kontakt mit endometrialen Oberflächen treten dürften ([15]; • Abb. 1). 
Bei der IVF und ICSI kommt jedoch kein Kontakt zwischen Ejakulat und Endometrium zustande, zumal hier im Regelfall von Geschlechtsverkehr abgeraten wird. Die niedrige Implantationsrate intakter Embryonen nach IVF oder ICSI lässt sich somit möglicherweise durch die fehlende physiologische Stimulation des Endometriums durch das Seminalplasma erklären. Gestützt werden diese Vermutungen durch verschiedene humanbiologische Studien.

Die Inkubation von endometrialen Epithelzellen führte in vitro zu einer Stimulation der Expression von IL-1 $\beta$, IL-6 und LIF [11]. Dieser Effekt war maximal in Epithelzellen ausgeprägt, die in der 2. Zyklushälfte gewonnen wurden. Die Expressionszunahme dieser Zytokine wird insbesondere durch TGF- $\beta$ und IL-8 induziert, die im Seminalplasma in hohen Konzentrationen zu finden sind [11]. In einer erweiterten Untersuchung unter Anwendung von RNA-Arrays wurde das gesamte Spektrum an endometrialen epithelialen Faktoren untersucht, die durch Seminalplasma induziert werden. Von 55.000 untersuchten Genen wurde die Expression von 133 Faktoren stimuliert und von 12 Faktoren inhibiert ([18]; M. von Wolff, persönliche Mitteilung).

Da In-vitro-Experimente keinen Effekt von Prostaglandinen auf die Expressionen epithelialer Zytokine nachweisen konnten (M. von Wolff, persönliche Mitteilung), ist anzunehmen, dass die Wirkung des Seminalplasmas im Wesentlichen auf dessen Proteinen beruht. Im klinischen Kontext ist es von Bedeutung, dass der stimulierende Effekt von Seminalplasma in vitro nicht von der Qualität des Spermiogramms abhängig ist [26].

Neben diesen grundlagenwissenschaftlichen Hinweisen für einen Effekt von Seminalplasma auf die endometriale Funktion liegen diverse klinische Studienergebnisse vor, die eine positive Wirkung von Seminalplasma stützen.

Bellinge et al. [2] führten in einer prospektiv randomisierten Studie bei $58 \mathrm{~Pa}$ tientinnen Ejakulat wenige Stunden nach der Follikelpunktion in die Vagina ein und fanden eine Implantationsrate von $53 \%$ im Vergleich $\mathrm{zu} 23 \%(\mathrm{p}<0,01)$ in einer unbehandelten Kontrollgruppe $(n=56)$. Coulam et al. [5] führten eine placebokontrol-

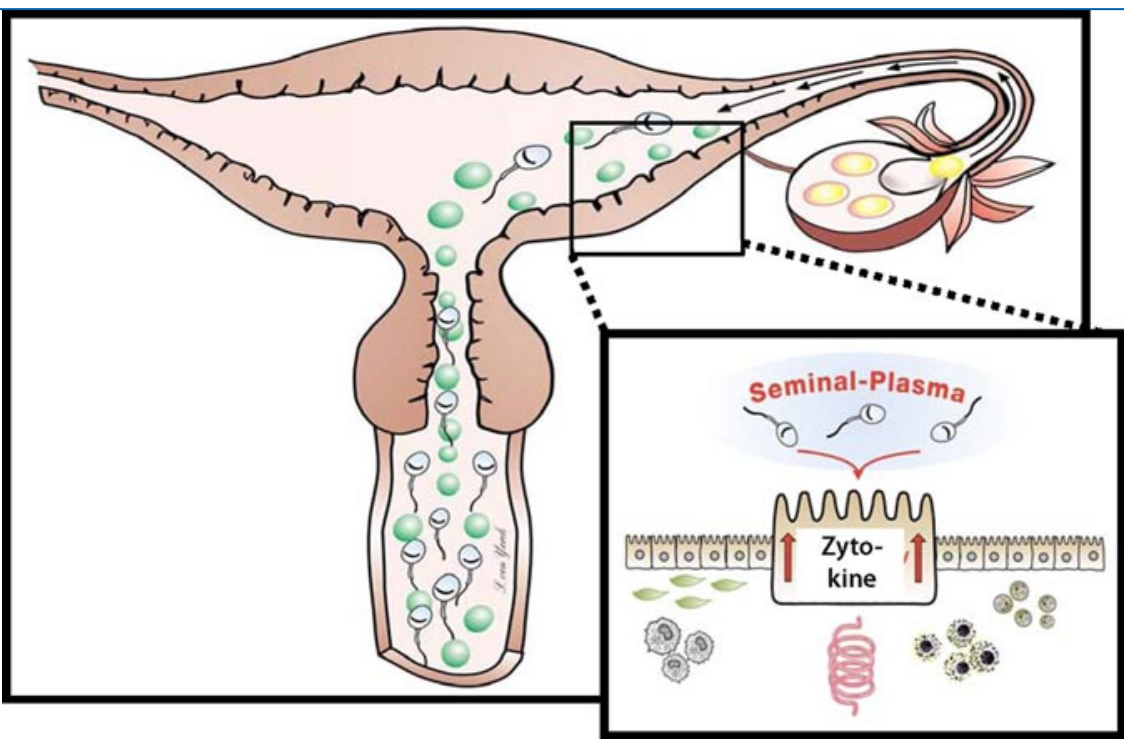

Abb. $1 \Delta$ Modell der physiologischen und therapeutischen Wirkung von Seminalplasma auf das Endometrium. Seminalplasma wird durch die uterine Peristaltik und durch Kapillarkräfte in das Cavum uteri gesogen, wo es am Endometrium die Expression von Zytokinen induziert

lierte, randomisierte Studie durch und legten Kapseln mit $(n=44)$ und ohne Seminalplasma $(n=43)$ im Verlauf eines Jahres regelmäßig in die Vagina ein. Die Implantationsrate betrug $80 \%$ in der Verumgruppe im Vergleich zu 67\% in der Placebogruppe.

In einer aktuellen, prospektiv randomisierten, placebokontrollierten Doppelblindstudie wurde Seminalplasma vom Partner der Patientin oder Placebo $(\mathrm{NaCl})$ direkt nach der Follikelpunktion in die Vagina und in die Zervix injiziert [27]. Die klinische Schwangerschaftsrate betrug bei den 67 Verum-behandelten Patientinnen $37,3 \%$ im Vergleich zu 25,7\% in der Placebo-behandelten Kontrollgruppe. Der Unterschied war bei der untersuchten Zahl behandelter Patientinnen jedoch noch nicht statistisch signifikant.

\section{Seminalplasmatische Proteine modellieren die endometriale Funktion und können die Implantationsrate verbessern}

Zusammenfassend liegen somit sowohl grundlagenwissenschaftliche als auch klinische Daten vor, die darauf hindeuten, dass seminalplasmatische Proteine die endometriale Funktion modellieren und somit die Implantationsrate verbessern können (• Abb. 1). Ein eindeutiger Beweis im Sinne einer großen, prospektiv randomisierten, placebokontrollierten
Doppelblindstudie mit einem eindeutig statistisch signifikanten Ergebnis steht jedoch noch aus.

\section{Geschlechtsverkehr bei der IVF/IVSI-Behandlung}

Die Hinweise für eine Steigerung der Schwangerschaftsrate durch die Interaktion von Seminalplasma mit dem Endometrium werfen die Frage auf, ob Geschlechtsverkehr ähnliche Effekte wie eine Behandlung mit Seminalplasma induzieren kann.

$\mathrm{Zu}$ dieser Fragestellung liegt jedoch nur eine randomisierte klinische Studie vor [23]: 478 IVF-Zyklen wurden randomisiert, sodass die Patientinnen entweder am Tag des Embryotransfers ( \pm 2 Tage) Geschlechtsverkehr (GV) hatten oder abstinent blieben (Kontrolle). Die Schwangerschaftsrate in der GV-Gruppe betrug 23,6\% und in der Kontrollgruppe 21,2\%. Dieser Unterschied war nicht statistisch signifikant. Allerdings war die Zahl vitaler Embryonen (11/10o transferierter Embryonen) in der GV-Gruppe signifikant höher $(\mathrm{p}=\mathrm{o}, 036)$ als in der Kontrollgruppe (7,7/10o transferierter Embryonen).

Gemäß dieser Studie vermag Geschlechtsverkehr auch eine geringe (nicht signifikante) Steigerung der Implantationsrate zu bewirken. Diese Steigerung scheint jedoch geringer zu sein als bei einer Behandlung mit Seminalplasma zum 
Zeitpunkt der Follikelpunktion. Aufgrund der unterschiedlichen Studiendesigns und der überwiegend nicht signifikanten Ergebnisse ist eine abschließende Bewertung jedoch noch nicht möglich.

\section{Fazit für die Praxis}

Bei alleiniger männlicher Infertilität besitzt das Spermiogramm nur wenig diagnostische Aussagekraft. Es kann durchaus gerechtfertigt sein, auch bei pathologischen Spermiogrammen eine IUI als erste Behandlungsoption anzubieten. Mit der neuen IMSI-Technik scheinen selbst aussichtslose Fälle männlicher Unfruchtbarkeit behandelbar zu werden. Geschlechtsverkehr zur Spermagewinnung, eine kurze Abstinenzzeit und Spermienabgabe zu Hause können als einfache Maßnahmen die Samenqualität von Kinderwunschpatienten entscheidend verbessern. Seminalplasma scheint mithilfe von Zytokinen das Endometrium positiv beeinflussen zu können und somit die Implantationswahrscheinlichkeit des Embryos zu erhöhen. Da bei reproduktionsmedizinischen Behandlungen meist kein Seminalplasma übertragen wird, könnte man den Patienten Geschlechtsverkehr um die Zeit des Embryotransfers empfehlen.

\section{Korrespondenzadresse \\ Dr. M. Nitzschke}

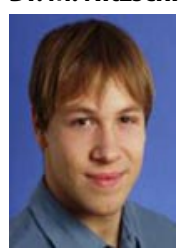

Abteilung Gynäkologische Endokrinologie und Reproduktionsmedizin, Universitätsklinik für Frauenheilkunde Effingerstraße 102, 3010 Bern, Schweiz markus.nitzschke@insel.ch

Interessenkonflikt. Der korrespondierende Autor gibt an, dass kein Interessenkonflikt besteht.

\section{Literatur}

1. Baker RR (1997) Copulation, masturbation, and infidelity. New Aspects of Human Ethology. Springer, Plenum Press, New York, USA

2. Bellinge BS, Copeland CM, Thomas TD et al (1986) The influence of patient insemination on the implantation rate in an in vitro Fertilization and embryo transfer program. Fertil Steril 46:252-256

3. Berkovitz A, Eltes F, Lederman $\mathrm{H}$ et al (2006) How to improve IVF-ICSI outcome by sperm selection. Reprod Biomed Online 12:634-638
4. Clarke RN, Klock SC, Geoghegan A, Travassos DE (1999) Relationship between psychological stress and semen quality among in-vitro fertilization patients. Hum Reprod 14:753-758

5. Coulam CB, Stern JJ (1995) Effect of seminal plasma on implantation rats. Early Pregnancy 1:33-36

6. David S, Guzick DS, Overstreet JW et al (2001) Sperm morphology, motility, and concentration in fertile and infertile men. N Engl J Med 345:13881393

7. De Vos A, Van De Velde H, Joris H et al (2003) Influence of individual sperm morphology on fertilization, embryo morphology, and pregnancy outcome of intracytoplasmic sperm injection. Fertil Steril 79:42-48

8. Elzanaty S, Malm J (2007) Effects of ejaculation-toanalysis delay on levels of markers of epididymal and accessory sex gland functions and sperm motility. J Androl 28:847-852

9. Elzanaty S, Malm J (2008) Comparison of semen parameters in samples collected by masturbation at a clinic and at home. Fertil Steril 89:1718-1722

10. Franco JG Jr, Baruffi RLR, Mauri AL et al (2007) What's the signification of nuclear vacuoles in human sperm? RBM Online RB 3286

11. Gutsche $S$, von Wolff M, Strowitzki T, Thaler CJ (2003) Seminal plasma induces mRNA expression of IL-1 $\beta$, IL- 6 and LIF in endometrial epithelial cells vitro. Mol Hum Reprod 9:785-791

12. Hazout A, Dumont-Hassan $M$, Junca AM et al (2006) High-magnification ICSI overcomes paternal effect resistant to conventional ICSI. Reprod Biomed Online 12:19-25

13. Horcajades JA, Riesewijk A, Polman J et al (2005) Effect of controlled ovarian hyperstimulation in IVF on endometrial gene expression profiles. Mol Hum Reprod 11:195-205

14. Ibérico G, Vioque J, Ariza N (2004) Analysis of factors influencing pregnancy rates in homologous intrauterine insemination. Fertil Steril 81(5):13081313

15. Kunz G, Beil D, Deiniger H et al (1997) The uterine peristaltic pump. Normal and impeded sperm transport within the female genital tract. Adv Exp Med Biol 424:267-277

16. Levitas E, Lunenfeld E, Weiss $\mathrm{N}$ et al (2005) Relationship between the duration of sexual abstinence and semen quality: analysis of 9,489 semen samples. Fertil Steril 83(6):1680-1686

17. Ombelet $W$, Vandeput $H$, Van de Putte $G$ et al (1997) Intrauterine insemination after ovarian stimulation with clomiphene citrate: predictive potential of inseminating motile count and sperm morphology. Hum Reprod 12:1458-1463

18. Pinheiro MR, Capp E, Strowitzki T, von Wolff M (2007) The effect of selected genes involved in implantation. Hum Reprod 122 (Suppl 1):P-493

19. Robertson SA, Roberts CT, Farr KL et al (1999) Fertility impairment in granulocyte-macrophage colony-stimulating factor-deficient mice. Biol Reprod 60:251-261

20. Robertson SA, O'Connell AC, Hudson SN, Seamark RF (2000) Granulocyte-macrophage colony-stimulating factor (GM-CSF) targets myeloid leukocytes in the uterus during the post-mating inflammatory response in mice. J Reprod Immunol 46:131154

21. Robertson SA, Sharkey DJ (2001) The role of semen in induction of maternal immune tolerance in pregnancy. Semin Immunol 13:243-254

22. Sofikitis NV, Miyagawa I (1993) Endocrinological, biophysical, and biochemical parameters of semen collected via masturbation versus sexual intercourse. J Androl 14:366-373
23. Tremellen KP, Valbuena D, Landeras J et al (2000) The effect of intercourse on pregnancy rates during assisted human reproduction. Hum Reprod 15:2653-2658

24. Tur-Kaspa I, Maor Y, Levran D et al (1994) How often should infertile men have intercourse to achieve conception? Fertil Steril 62:370-375

25. Van Voorhis BJ, Barnett M, Sparks AE et al (2001) Effect of the total motile sperm count on the efficacy and cost-effectiveness of intrauterine insemination and in vitro fertilization. Fertil Steril 75:661668

26. von Wolff M, Nowak O, Pinheiro RM, Strowitzki T (2007) Seminal plasma - immunomodulatory potential in men with normal and abnormal sperm count. Eur J Obstet Gynecol Reprod Biol 14:73-78

27. von Wolff $M$, Rösner S, Thöne C et al (2008) Intravaginal and intracervical application of seminal plasma in IVF/ICSI-treatment cycles - a double blind placebo-controlled randomised pilot study. Fertil Steril 2 (Epub ahead of print)

28. Wainer R, Albert M, Dorion A et al (2004) Influence of the number of motile spermatozoa inseminated and of their morphology on the success of intrauterine insemination. Hum Reprod 19:2060-2065

29. World Health Organization (1999) Laboratory manual for human semen and sperm cervical mucus interaction, 4th edn. Cambridge University Press, Cambridge

30. Zavos PM, Goodpasture JC (1989) Clinical improvements of specific seminal deficiencies via intercourse with a seminal collection device versus masturbation. Fertil Steril 51:190-193 